\title{
Experimental Optimization of Exposure Index and Quality of Service in WLAN networks
}

\author{
David Plets, Günter Vermeeren, Eli De Poorter, Ingrid Moerman, Luc Martens, Wout Joseph \\ Ghent University/iMinds, Dept. of Information Technology, Gaston Crommenlaan 8, B-9050 Gent, Belgium \\ david.plets@intec.ugent.be
}

\begin{abstract}
exposure, exposure index, WLAN, WiFi, optimization, genetic algorithm, uplink, downlink, power density, RF, radiofrequency, population, testbed, path loss, experimental, power control.
\end{abstract}

\section{INTRODUCTION}

In order to ensure the best mobile connectivity to users, wireless networks have become omnipresent in today's society. Wireless Local Area Networks (WLANs) provide a high-bandwidth connectivity and are compatible with common wireless devices such as smartphone or tablets. They are mostly used to cover indoor environments and are useful for operators in a sense that they also often serve as an offloading mechanism for macrocell networks. The ever-increasing use of wireless networks has also caused an increase in the concerns about possible health effects of exposure to electromagnetic field (EMF) radiation. To that end, radio-frequency (RF) exposure has been subjected to national and international limits. The International Commission on Non-Ionizing Radiation Protection (ICNIRP) Guidelines [1] has defined limitations for the Specific Absorption Rate (SAR) for RF sources at frequencies between $100 \mathrm{kHz}$ and $6 \mathrm{GHz}$. ICNIRP also define reference levels that limit incident field strength to the level inducing an exposure compliant with basic restrictions. However, these restrictions are based on worstcase assumptions and do not assess the actual exposure of users. Realistic assessment of the exposure of users is approached in different ways. Firstly, the incident field strength or power density characterizes the exposure due to the radiation of base stations. It only accounts for far-field exposure due to broadcast or downlink traffic and comprises most of the experimental research carried out so far [2], [3], [4], [5], [6]. Secondly, the morphology of the considered user [7], [8], [9], [10] is added by calculating the SAR [11], which characterizes the RF energy absorption in the human body. This formulation also allows assessing the near-field exposure due to the uplink of the user device: in [12], [13], a joint assessment is made of the wholebody SAR due to both uplink and downlink traffic of a user. Thirdly, by multiplying the SAR with the actual exposure time, the exposure dose of a user can be calculated [12]. It is clear that these three metrics are focused on exposure assessment of individuals. Therefore, the authors of [14] have formulated the Exposure Index (EI) metric, which characterizes the average SAR of a certain population within a given time frame. This allows comparison of different wireless deployment configurations with respect to their resulting exposure of a large set of users. Although the EI has already been calculated for given deployment, no algorithms are available yet, that optimize the wireless network configuration for a minimal EI, within given Quality of Service (QoS) and/or cost restrictions. Analogously to the three aforementioned exposure assessment approaches, former optimization studies were limited to minimization of the electric-field strength [15] or a SAR/dose minimization [12].

In this paper, an algorithm will be presented that implements the detailed EI formula and optimizes the wireless configuration for a minimal EI of the considered population, based on a number of inputs (e.g., maximal number of base stations allowed, number of users, user traffic characteristics,...). Also exposure due to uplink activity of other users is accounted for, since it can significantly contribute to the total exposure when the user device does not implement power control or when the wireless connection quality is bad [16], [17]. Although the presented algorithm is applicable to both indoor and outdoor wireless networks, it will here be applied to WiFi networks in indoor environments, as exposure from WLANs can be significant [2]. A comparison with traditional deployments will be made. This paper is the first to present an EI optimization algorithm. It comes forward to the European Union's need for low-EMF system designs that was formulated in its Seventh Framework Program (FP7, ICT Call 8) [18]. This target outcome specifies a clear need for new network topologies and management that reduce EMF levels without compromising the users QoS. Moreover, unlike in previous exposure optimization research using E-models, the optimization will be performed based on an experimental determination of the powers and power densities in the network, hereby canceling all uncertainty with respect to the correctness of the EI.

The remainder of the paper is structured as follows. In Section II, the methodology of the algorithm is presented, discussing in detail the architecture of the solution, the environment in which the EI is calculated, the EI and how it is calculated, the genetic algorithm (GA) used to find the optimal deployment, and the application scenario for which the algorithm will be tested. Section III presents and discusses the results and the paper's main findings are summarized in Section IV. 


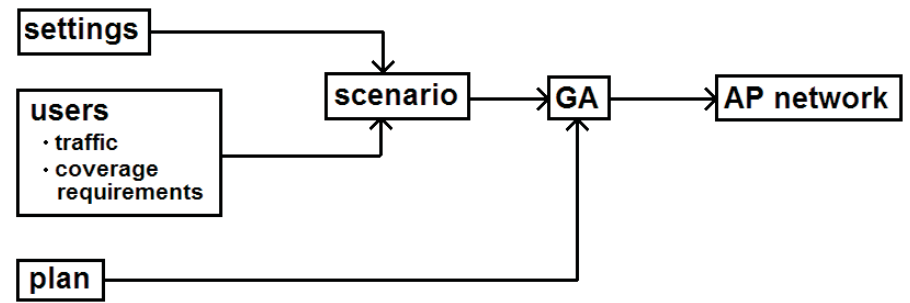

Fig. 1: Architecture of the EI optimization algorithm.

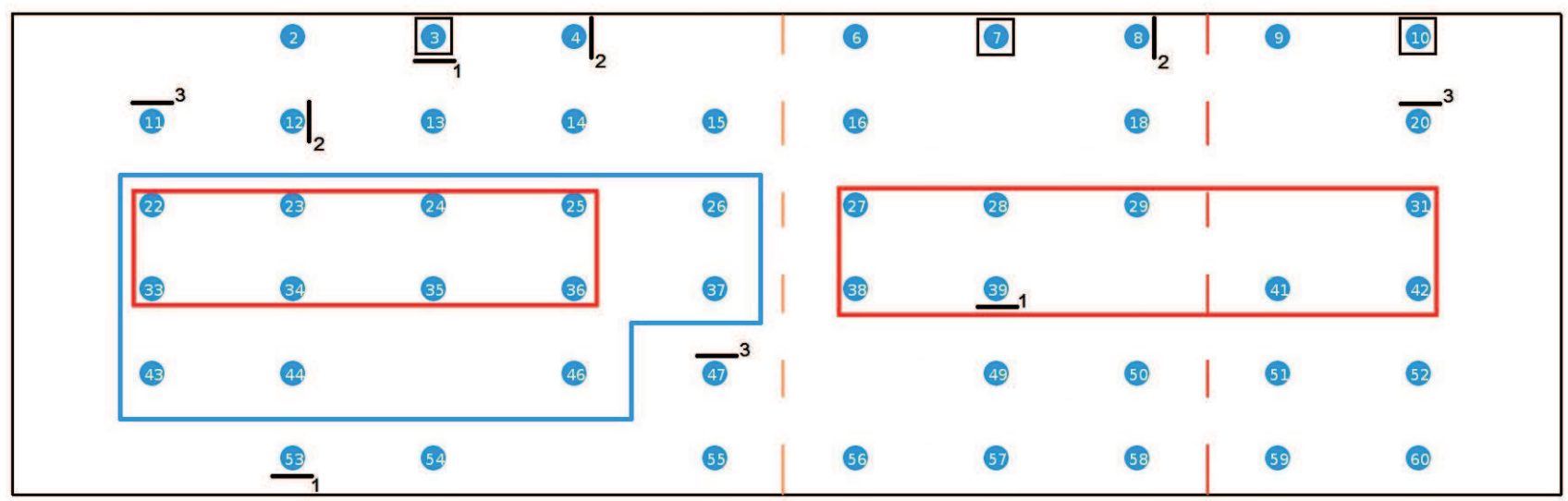

Fig. 2: Experimentation environment $(66 \mathrm{~m} \mathrm{x} 20.5 \mathrm{~m})$ with indication of the $60 \mathrm{WiFi}$ nodes. The dashed vertical lines indicate assumed walls to divide the room into three adjacent rooms (only for scenario 1). APs in the reference configuration are indicated by rectangles. The chosen APs for the optimization with 3 APs for scenarios 1, 2, and 3 are indicated with a lower line, right line, and upper line, respectively, with indication of the scenario number. Users in scenario 1 are within the blue rectangle, users in scenarios 2 and 3 are within the two red rectangles.

\section{Methodology}

\section{A. Solution}

The core of the optimization algorithm is a genetic algorithm, which will be explained in more detail in Section II-E. The algorithm takes as input the physical ground plan of the environment and the wireless scenario. The latter is determined by the settings (e.g., maximal number of APs allowed, power control on/off,...) and by the users. The set of users and their location is defined, the coverage requirement they have, and the (estimated) characteristics of their traffic: the time fraction of the actual traffic activity and the type of activity (e.g., surfing, video call,...), which impacts the duty cycle of the traffic. The output of the algorithm is a wireless network configuration with a set of active access points with a certain Effective Isotropically Radiated Power (EIRP).

\section{B. Experimentation environment}

All test scenarios will be experimentally assessed inside an open pseudo-shielded testbed environment (w-iLab.t) in Ghent, Belgium. It consists of 60 nodes that are identified by a number (1-60). All nodes are mounted in an open room (66 m x $20.5 \mathrm{~m})$ in a grid configuration with an x-separation of $6 \mathrm{~m}$ and a y-separation of $3.6 \mathrm{~m}$. Fig. 2 shows the ground plan of the living lab with an indication of the location of the nodes (blue). Each node has two WiFi interfaces (Sparklan WPEA-110N/E/11n mini PCIe 2T2R chipset: AR9280) and to each WiFi card, two antennas are connected (2x2 MIMO is supported). Furthermore, an RM090 sensor node and a USB2.0 Bluetooth interface (Micro CI2 - v3.0 EDR) are incorporated into each node. The testbed allows sending and receiving WiFi packets between any two nodes and thus allows a realistic assessment of actual exposure values in the network.

\section{Exposure index}

Recently, attempts have been made to characterize the exposure impact of wireless network deployments, e.g., in [16]. In [14], the exposure index EI was proposed, a new metric accounting for the exposure induced by base station antennas as well as wireless devices. 


$$
E I^{S A R}=\frac{1}{T} \sum_{t}^{N_{T}} \sum_{p}^{N_{p}} \sum_{e}^{N_{R}} \sum_{r}^{N_{R}} \sum_{c}^{N_{C}} \sum_{l}^{N_{L}} \sum_{\text {pos }}^{N_{\text {pos }}} f_{t, p, e, r, c, l, p o s}\left[\sum_{u}^{N_{U}}\left(d^{U L} \bar{P}_{T X}\right)+d^{D L} \bar{S}_{\text {inc }}^{D L}+d^{U L, \text { other }} \bar{S}_{\text {inc }}^{U L, \text { other }}\right][W / k g]
$$

where $\mathrm{EI}^{\mathrm{SAR}}$ is the EI value, the average exposure of the population of the considered geographical area over the considered time frame T. SAR refers to whole-body SAR, organ-specific SAR, or localized SAR. $\mathrm{N}_{\mathrm{T}}$ is number of considered periods within the considered time frame (e.g., single day); $\mathrm{N}_{P}$ is the number of considered population categories; $\mathrm{N}_{\mathrm{E}}$ is the number of considered environments; $N_{R}$ is the number of considered RATs; $N_{C}$ is the number of considered cell types; $N_{L}$ is the number of considered user load profiles; $\mathrm{N}_{\text {pos }}$ is the number of considered postures; and $\mathrm{N}_{\mathrm{U}}$ is the number of considered usages with devices. $\overline{\mathrm{P}}_{\mathrm{TX}}$ is the mean TX power transmitted by users devices during period $\mathrm{t}$, in usage mode $\mathrm{u}$, connected to RAT r, in environment e. A TX power values map is given for the whole considered area and the average value is taken into account for EI evaluation; $\overline{\mathrm{S}}_{\mathrm{inc}}^{\mathrm{DL}}$ is the mean incident power density on the human body during period $t$, in environment $\mathrm{e}$, induced by all base stations of RAT $r$, cell type $c$. A distribution of the incident power density for the whole considered area is considered and the average value over this area is taken into account for the EI evaluation. $\overline{\mathrm{S}}_{\text {inc }}^{\mathrm{UL} \text {, other }}$ is the total incident power density on the human body during period $t$ in environment $\mathrm{e}$, induced by the wireless devices of all other users in the proximity that are connected to RAT $\mathrm{r}$ and cell type c. $\mathrm{d}^{\mathrm{UL}}(\mathrm{Ws} / \mathrm{kg}$ per $\mathrm{W}), \mathrm{d}^{\mathrm{UL} \text {,other }}\left(\mathrm{Ws} / \mathrm{kg}\right.$ per $\left.\mathrm{W} / \mathrm{m}^{2}\right)$, and $\mathrm{d}^{\mathrm{DL}}(\mathrm{Ws} / \mathrm{kg}$ per $\mathrm{W} / \mathrm{m}^{2}$ ) are normalized raw dose values for UL, DL from the user in the proximity, and DL from base stations and access points, respectively, all multiplied by time spent in the configuration; $f_{t, p, e, r, c, l, p o s}$ is the fraction of the total population that corresponds to population category $\mathrm{p}$, user load profile 1 , in posture pos, connected to RAT $\mathrm{r}$, for cell type $\mathrm{c}$, in environment e during time period $t$.

The way of formulating the EI using population fractions (as in eq. 1) is required, since the EI is often calculated over a very large geographic area with many users and over a large time frame, where uplink powers and downlink power densities are not known for each distinct individual and his usages. Therefore, the population is divided into fractions consisting of people in the same environment, using the same posture,.., all transmitting (receiving) similar uplink (downlink) powers (power densities). In the indoor scenarios considered here, it will be assumed that the location of the considered users $u s e r$ in the population pop and the transmitted powers and received power densities of each of them can be exactly determined, so the EI can be considered as a composition of each user's specific exposure, with his power (density), usage time and duration, device, posture, usage,... always known. Using a summation over each user's EI allows removing the use of fractions $f$, and abolishes the need to distinguish between time period $t$, environment $e$ or user load profiles $l$. Here, the EI will be considered for WiFi-only traffic and one posture pos, meaning that also the summation over $r$, $c$, and pos can be removed. The use of the average power densities $\overline{\mathrm{S}}_{\text {inc }}$ and $\overline{\mathrm{S}}_{\mathrm{inc}}^{\mathrm{UL} \text {,other }}$ can then also be replaced by a summation over the average contributions of each specific base station (DL) and of the other users' UL, respectively. Eq. 2 then transforms to

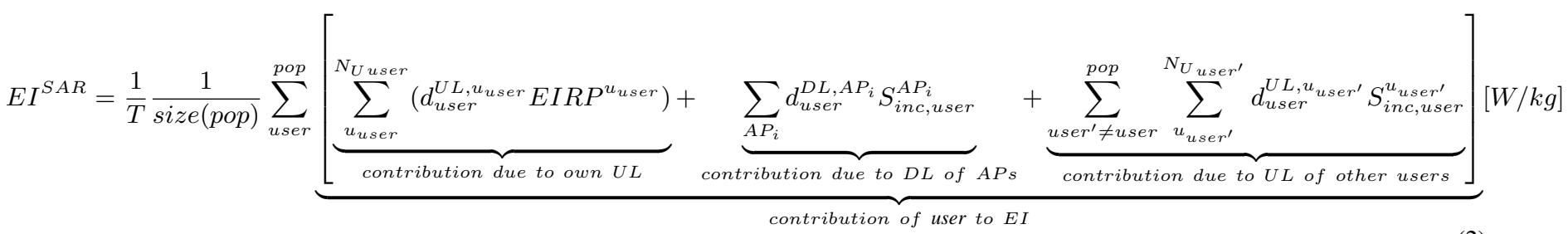

where $\mathrm{EI}^{\mathrm{SAR}}[\mathrm{W} / \mathrm{kg}]$ is the EI value, the average exposure over all users user within the population pop over the considered time frame T [s]. $u_{\text {user }}\left(\right.$ or $u_{u s e r^{\prime}}$ ) is a specific usage of user (or user'); $N_{U \text { user }}\left(\right.$ or $N_{U \text { user }}$ ) is the set of all usages of user user (or user'). Usages are e.g., using a laptop on the table for surfing, using a laptop on the lap for a Skype video call, or no usage at all (= no uplink traffic). The sum of the durations of all usages of a certain user must be equal to the total considered time frame T. $d_{\text {user }}^{U L, u_{u s e r}}\left(\mathrm{Ws} / \mathrm{kg}\right.$ per W), $d_{\text {user }}^{D L, A P_{i}}\left(\mathrm{Ws} / \mathrm{kg}\right.$ per $\left.\mathrm{W} / \mathrm{m}^{2}\right)$, and $d_{\text {user }}^{U L, u_{u s e r}}\left(\mathrm{Ws} / \mathrm{kg}\right.$ per $\left.\mathrm{W} / \mathrm{m}^{2}\right)$ are normalized raw dose values at the location of user for UL usage $u_{u s e r}$ (source in near-field of user), for DL traffic from access point $A P_{i}$ (source in far-field of user), and for UL usage $u_{u s e r}$ (source in far-field of user), all multiplied by the time spent in the configuration. EIRP ${ }^{u_{u s e r}}[\mathrm{~W}]$ is the EIRP of user in the considered usage $u_{u s e r}$, scaled with the duty cycle corresponding to $u_{\text {user. }} S_{\text {inc,user }}^{A P_{i}}\left[\mathrm{~W} / \mathrm{m}^{2}\right]$ is the induced power density by access point $A P_{i}$ at the location of $u s e r$, and $S_{\text {inc,user }}^{u_{u s e r}}\left[\mathrm{~W} / \mathrm{m}^{2}\right]$ the induced power density by $u s e r^{\prime}$ 's usage $u_{u s e r}$ at the location of user.

$$
d_{\text {user }}^{U L, u_{u s e r}}\left(\frac{W s}{k g} / W\right)=T D^{U L, u_{u s e r}}(s) \cdot S A R_{\text {ref,user }}^{N F, \text { user }}\left(\frac{W}{k g} / W\right),
$$

with $T D^{U L, u_{u s e r}}$ the time duration of UL usage $u_{u s e r}$ and $S A R_{r e f, u s e r}^{N F, \text { user }}$ the near-field (NF) reference SAR for $u s e r$ due to his uplink usage. 


$$
d_{\text {user }}^{D L, A P_{i}}\left(\frac{W s}{k g} / \frac{W}{m^{2}}\right)=T D^{D L, A P_{i}}(s) \cdot S A R_{\text {ref,user }}^{F F, A P_{i}}\left(\frac{W}{k g} / \frac{W}{m^{2}}\right),
$$

with $T D^{D L, A P_{i}}$ the time duration of DL activity of $A P_{i}$ and $S A R_{r e f, u s e r}^{F F, A P_{i}}$ the far-field (FF) reference SAR for $u s e r$ due to $A P_{i}$ 's radiation.

$$
d_{\text {user }}^{U L, u_{u s e r^{\prime}}}\left(\frac{W s}{k g} / \frac{W}{m^{2}}\right)=T D^{U L, u_{u s e r^{\prime}}}(s) \cdot S A R_{r e f, u s e r}^{F F, u s e r^{\prime}}\left(\frac{W}{k g} / \frac{W}{m^{2}}\right),
$$

with $T D^{U L, u_{u s e r}}$ the time duration of the UL usage $u_{u s e r^{\prime}}$ of $u s e r^{\prime}$ and $S A R_{\text {ref,user }}^{F F, r^{\prime}}$ the far-field (FF) reference SAR for user due to the UL of $u s e r^{\prime}$.

$S_{\text {inc } \text {,user }}^{\text {source }}$ as in eq. (2) (with source $=A P_{i}$ or $u_{u s e r^{\prime}}$ ) is calculated as

$$
\mathrm{S}_{\text {inc,user }}^{\text {source }}=\frac{\left(\mathrm{E}_{\text {inc,user }}^{\text {source }}\right)^{2}}{\mathrm{Z}_{0}}=\frac{\left(\mathrm{E}_{\text {inc,user }}^{\text {source }}\right)^{2}}{120 \cdot \pi}=\frac{\left(\mathrm{E}_{\text {inc,user }}^{\text {source }}\right)^{2}}{377},
$$

$E_{\text {inc,user }}^{\text {source }}[\mathrm{V} / \mathrm{m}]$ is the incident electric-field strength at the location of user due to the access point $A P_{i}$ or the other user's usage $u_{u s e r}$, and with an assumed duty cycle of $100 \% . \mathrm{Z}_{0}$ is the free-space impedance, equal to $377 \Omega$. For WiFi, the actual duty cycle DC [-] of the traffic generated by the source [19] must also be accounted for, since it represents the relative transmission time of a signal. In WiFi, signals are not transmitted continuously and therefore the predicted power densities at $100 \%$ operation need to be multiplied by the duty cycle. When accounting for the duty cycle, eq. (6) can be rewritten as follows:

$$
S_{\text {inc,user }}^{\text {source }}=\frac{\left(E_{\text {inc,user }}^{\text {source }}\right)^{2} \cdot D C}{377},
$$

Finally, E $E_{\text {inc,user }}^{\text {source }}$ from eq. (7) is calculated as follows:

$$
\mathrm{E}_{\text {inc,user }}^{\text {source }}[\mathrm{V} / \mathrm{m}]=10^{\frac{\text { EIRP }^{\text {source }}-43.15+20 \cdot \log _{10}(\mathrm{f})-\mathrm{PL}_{\text {source, }}^{\text {scenario }}}{20}}[15],
$$

with $E I R P^{\text {source }}[\mathrm{dBm}]$ the EIRP of the source with a duty cycle of $100 \%, \mathrm{f}[\mathrm{MHz}]$ the frequency, and $P L_{\text {source }}^{\text {scenario }}$ [dB] the path loss between the source and the user in the considered scenario. The path loss values $P L_{\text {source, user }}$ are experimentally determined in the testbed.

\section{Scenario}

Three scenarios will be defined within the test environment of Fig. 2, for which the wireless deployment will be optimized in order to guarantee the lowest EI at each instant, while still providing the required QoS. For all three scenarios, adult users are considered, using a laptop on a table in front of them. For adult users, $S A R_{\text {ref, }}^{F F \text {,user }}$ (with source $=A P_{i}$ or $u s e r^{\prime}$ ) is $0.0049 \frac{\mathrm{W}}{\mathrm{kg}} / \frac{\mathrm{W}}{\mathrm{m}^{2}}$ [20], $S A R_{\text {ref, user }}^{N F, \text { iser }} 0.0027 \frac{\mathrm{W}}{\mathrm{kg}} / \mathrm{W}$ [20]. In WiFi, there is no power control mechanism by default, so the own laptop's transmit power $E I R P^{u_{u s e r}}$ from eq. (2) and the other users' laptop output power $E I R P^{u_{u s e r}}$ from eq. (8) will then be assumed fixed at $100 \mathrm{~mW}$ and $20 \mathrm{dBm}$ respectively, except when the usage is equal to no usage, where EIRP ${ }^{u s e r}$ will be $0 \mathrm{~W}$ and $E I R P^{u_{u s e r}}$ will be $-\infty$ (expressed in $\mathrm{dBm}$ ).

The first scenario assumes that the environment consists of three adjacent rooms, with inner walls indicated by the dashed lines in Fig. 2. The left dashed line indicates the location of a layered drywall with a penetration loss PenL of $2 \mathrm{~dB}$, the right dashed wall is a brick wall with an assumed PenL of $8.5 \mathrm{~dB}$. Since the testbed is an open environment without walls, the experimentally determined path losses $P L_{\text {source, }}^{\text {testber }}$ have to be adjusted to account for the path loss $P L_{\text {source, }}^{\text {scenario }}$ according to the scenario (see eq. (8)). To this end, the sum of the penetration losses PenL of the set of walls $W_{\text {source,user }}$ along the direct ray between source and user are added to the experimentally determined path losses $P L_{\text {source, }}^{\text {testber }}$ :

$$
P L_{\text {source,user }}^{\text {scenario }}=P L_{\text {source, } \text { user }}^{\text {testbed }}+\sum_{W}^{W_{\text {source, user }}} \operatorname{Pen} L_{W},
$$

with $\operatorname{Pen} L_{W}$ the penetration loss of wall $\mathrm{W}$ from the set $W_{\text {source, user. }}$ E.g., a total penetration loss of $10.5 \mathrm{~dB}$ is added to the experimentally determined path loss between node 2 and 10 (one wall with $2 \mathrm{~dB}$ and one with $8.5 \mathrm{~dB}$ attenuation along the direct ray). The scenario further assumes that 13 people are present in the left room (at locations 22, 23, 24, 25, 26, 33, 34, 35, 36, 37, 43, 44, 46, see Fig. 2) following an online course of one hour under the form of a 1080p Youtube video (usage type). A physical data rate of $\mathrm{TP}_{\mathrm{DL}}^{\mathrm{req}} 54 \mathrm{Mbps}$ (corresponding to a required receiver power of $-68 \mathrm{dBm}[21]$ ) is required to allow the best capacity and best quality of service for the users. This wireless activity corresponds to a downlink duty cycle of $10.69 \%$ for one user [19]. Also based on [19], we assume that an access point's total duty cycle is calculated as the sum of the duty cycles due to the activity of each user that is connected to that specific access point; e.g., three users simultaneously watching 
a 1080p Youtube video via a certain AP, are assumed to cause that AP to have a duty cycle of $3 \cdot 10.69 \%=32.07 \%$. Users are considered to always connect to the access point that delivers the highest received power. This scenario is assumed to be a downlink-only scenario, so no uplink traffic is considered here.

The second scenario assumes 16 operators in a large call center that extends over the entire environment in Fig. 2, meaning that an open space is assumed without any inner walls. Users are located at 22, 23, 24, 25, 33, 34, 35, 36 and at 27, 28, 29, $31,38,39,41,42$, see Fig. 2. It is assumed that the operators use Voice-over-IP with their laptop. Hence, for each of the users a duty cycle of $0.8 \%$ is assumed for both uplink and downlink voice traffic, based on the measured duty cycles for 'Skype voice' at $54 \mathrm{Mbps}$ in [19]. While scenario 1 is a downlink-only scenario, scenario 2 jointly considers downlink and uplink. The third scenario explores a future deployment where WiFi with power control is considered. Recently, efforts have been made towards a standardization of power control in WiFi system, for lower interference, a lower energy consumption, and a lower human exposure [22], [23]. The third scenario will be defined equal to the second scenario, but with added uplink power control. This future scenario will allow comparing the impact of power control on the EI and on the optimal AP configuration. It will be assumed that the client adjusts its power $\mathrm{P}_{\text {client }}^{\mathrm{Tx}}$ in order to deliver the required received power $\mathrm{P}_{\mathrm{AP}}^{\text {req }}$ at the access

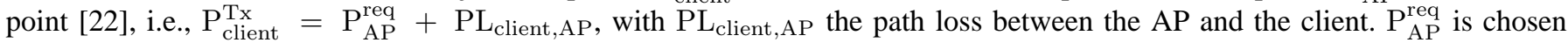
at $-68 \mathrm{dBm}$, aiming for an optimal connection with an uplink throughput of $54 \mathrm{Mbps}$.

It is clear that these three scenarios will each correspond to a different EI-optimal WiFi deployment. Based on the inputted scenario, the engine (see Fig. 1) will accordingly design the wireless network configuration that provides the required QoS with a minimal EI. All three scenarios will be optimized in two ways: without any restriction on the number of deployed APs and with a restriction to at most three deployed APs. Table I summarizes all parameter settings for the three considered scenarios.

TABLE I: Parameters settings for the three scenarios

\begin{tabular}{|c|c|c|}
\hline & SCENARIO 1 & SCENARIO 2 \\
\hline power control & no & yes \\
\hline user locations & $\begin{array}{r}22-23-24-25-26-33-34 \\
35-36-37-43-44-46\end{array}$ & $\begin{array}{l}22-23-24-25-33-34-35-36 \\
27-28-29-31-38-39-41-42\end{array}$ \\
\hline $\mathbf{T}$ & $3600 \mathrm{~s}$ & 8 hours \\
\hline usage & YouTube $1080 p$ & VoIP call \\
\hline TD ${ }^{\mathrm{DL}, \mathrm{AP}}$ & $0.9 \mathrm{~T}$ & $0.5 \mathrm{~T}$ \\
\hline TDUL,usage & $0 \mathrm{~T}$ & $0.5 \mathrm{~T}$ \\
\hline DC $_{\text {DL }}$ (peruser) & $10.69 \%$ & $0.8 \%$ \\
\hline $\mathrm{DC}_{\mathrm{UL}}$ & N/A & $0.8 \%$ \\
\hline $\mathbf{T P}_{\mathrm{DL}}^{\text {req }}$ & \multicolumn{2}{|c|}{$54 \mathrm{Mbps}(-68 \mathrm{dBm})$} \\
\hline $\mathbf{E I R P}_{\min }-$ EIRP $_{\text {max }}$ & \multicolumn{2}{|c|}{$0-20 \mathrm{dBm}$} \\
\hline $\mathbf{S A R}_{\text {ref }}^{\mathrm{UL}}$ & \multicolumn{2}{|c|}{$0.0027 \frac{\mathrm{W}}{\mathrm{kg}} / \mathrm{W}$ (adult, sitting, laptop on table) } \\
\hline $\mathbf{S A R}_{\text {ref }}^{\mathrm{DL}}$ & \multicolumn{2}{|c|}{$0.0049 \frac{\mathrm{W}}{\mathrm{kg}} / \frac{\mathrm{W}}{\mathrm{m}^{2}}$ (adult, sitting) } \\
\hline
\end{tabular}

\section{E. Genetic algorithm}

As shown in Fig. 1, a GA is developed to find the wireless configuration that corresponds with a minimal EI for a given scenario. A flow graph of the GA itself is shown in Fig. 3. The solution that is obtained with the GA will be compared with a reference solution, i.e., a traditional planning where one AP per room is deployed at an EIRP of $20 \mathrm{dBm}$, more concretely, at locations 3, 7, and 10 respectively. Logically, user locations are excluded as possible AP locations in the optimization process. Each individual in the population corresponds to a network deployment solution and is characterized by an array containing the EIRPs of the respective APs: each AP corresponds to a gene of a solution. Mathematically, each individual solution $X_{i}$, $\mathrm{i}=1$... population_size is represented by an $\mathrm{N}$-dimensional vector, where $\mathrm{N}$ represents the number of APs in the network, here equal to 60 (see Fig. 2).

$$
X_{i}=\left(X_{i, 1}, X_{i, 2}, \ldots, X_{i, N}\right)
$$

with $X_{i, j}, \mathrm{j}=1 \ldots \mathrm{N}$, a gene of solution $X_{i}$, representing either an integer value between $E I R P_{\min }$ and $E I R P_{\max }($ when switched on) or -1000 (when switched off):

$$
X_{i, j} \in\left(\left[E I R P_{\min }, E I R P_{\max }\right] \cap \mathbb{Z}\right) \cup\{-1000\}
$$

The GA starts with an initiation phase during which the scenario parameters are set as in Table II-D. After the initiation phase, a starting population of size population_size is built: each selectable AP is either switched off (probability of 90\%) or is assigned a random EIRP between $E I R P_{\min }$ and $E I R P_{\max }$ (probability of $10 \%$ ). Then, the population evolves over a number of generations equal to number_of_generations. Each generation consists of the following consecutive steps:

- Sorting - sort all previous individuals (=solutions) by their fitness value 


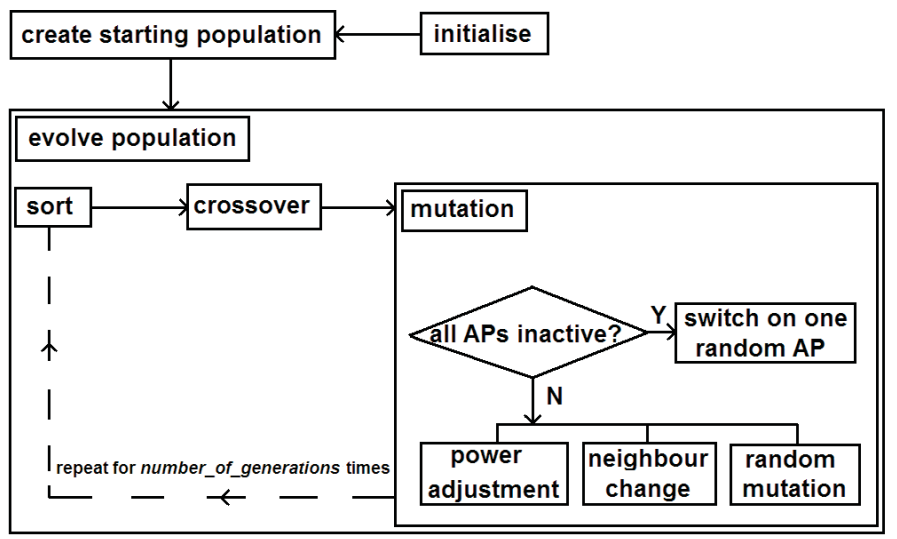

Fig. 3: Flow graph of genetic algorithm for network optimization.

- Crossover - from the previous population, a new population is created, whereby the first (best) elitism_size individuals from the previous population are transferred unchanged to the new population (elitism principle). The other population_size - elitism_size new solutions are child solutions, obtained from a crossover operation between two individuals, each chosen as the fittest individual out of a set of 5 random individuals from the population of the previous generation. Each child gene is inherited from either one of the corresponding parent genes, with equal probability. The newly created child solution is added to the new population.

- Mutation - all individuals in the obtained new population are mutated. If the mutation has a better fitness than the original individual, the original individual in the population is replaced by the mutated individual. The mutation of an individual is executed as follows:

- if all APs are inactive, one random AP is switched on with a random EIRP between $E I R P_{\min }$ and $E I R P_{\max }$

- else, three possible mutations are executed, each with a chance of $1 / 3$

* power adjustment - if the network provides a sufficient coverage to all users, the power of a random active AP is lowered with $1 \mathrm{~dB}$ (or switched off when its power is equal to $\mathrm{EIRP}_{\min }$ ), otherwise the power of a random AP is increased with $1 \mathrm{~dB}$ (when the AP is active with an EIRP smaller than $\mathrm{EIRP}_{\max }$ ) or switched on (when the AP is inactive).

* neighbour change - the EIRP of a random active AP is interchanged with that of an adjacent AP (see Fig. 2)

* random mutation - the EIRP of each AP (gene) of a solution (individual) is adjusted with a probability equal to mutation_rate: the change comprises switching off (probability of 90\%) or assigning a random EIRP between $E I R P_{\min }$ and $E I R P_{\max }$ (probability of $10 \%$ )

The paper does not aim at the development of an optimal optimization algorithm, so parameter values were chosen to obtain an optimal solution within an acceptable time.

TABLE II: Settings of genetic algorithm parameters

\begin{tabular}{|r|r|}
\hline population_size & 100 \\
\hline elitism_size & 10 \\
\hline mutation_rate & 0.025 \\
\hline number_of_generations & 30 \\
\hline
\end{tabular}

1) Fitness calculation: Each newly created individual is evaluated based on its fitness, expressed as the resulting EI value. First, it is calculated if the solution provides the required coverage and capacity to all of its connected users and if the number of active APs does not exceed the user-defined maximum. If this check is not passed, a default high EI value of $3000 \mathrm{~W} / \mathrm{kg}$ is returned to avoid being withheld as a possible solution. When the solution does pass the check, the EI fitness value is calculated as the average SAR value over all considered humans within the considered time frame. Each human's individual SAR value consists of three parts explained in eq. (2): the contribution due to all active APs, the contribution due the human's own wireless device, and the contribution due to other humans' wireless devices.

\section{RESULTS}

First, the path losses between all nodes in Fig. 2 will be experimentally determined and their lognormal behaviour will be tested. Then, the optimal wireless deployments for each of the three scenarios will be presented and compared. 


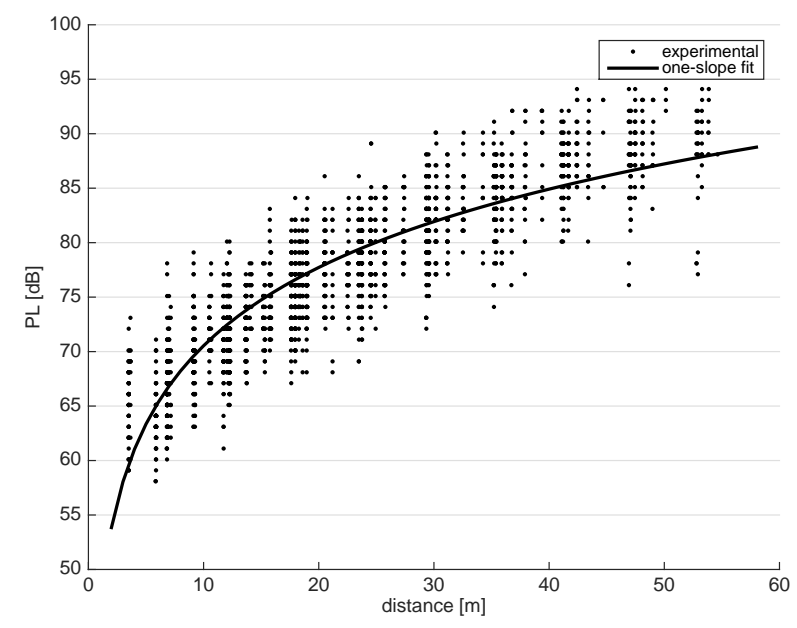

Fig. 4: Experimental path losses between all active nodes within the testbed and lognormal fit.

\section{A. Experimental path losses}

Fig. 4 shows the experimental path loss values in the testbed of Fig. 2, between all active nodes. The 2352 path loss samples are modeled using a one-slope log-distance model [24]:

$$
P L=P L_{0}+10 \cdot n \cdot \log (d)+\chi,
$$

where $\mathrm{d}(\mathrm{m})$ is the distance between the transmitter and the receiver, $\mathrm{n}$ is the path loss exponent, and $\mathrm{PL}_{0}$ is the path loss intercept at a distance of $1 \mathrm{~m}$. Furthermore, $\chi$ is the shadowing variation and has a standard deviation $\sigma$. A fit of the model parameters $\mathrm{PL}_{0}$ and $\mathrm{n}$ has been performed and the root-mean-square deviation of the measurement points was minimized with a linear regression fit. The parameters $\mathrm{PL}_{0}$ and $\mathrm{n}$ equal $46.66 \mathrm{~dB}$ and 2.39 respectively and the value of the standard deviation of the path loss samples around the model is $3.42 \mathrm{~dB}$. A Kolmogorov-Smirnov lognormality test at a significance level $\alpha=0.05$ proved the lognormal behaviour of the model. Although this shows that the fitted model could also be used for predicting the EI with a reasonable accuracy, the EI will here be calculated from the experimental PL values, since these will yield the exact EI value.

\section{B. EI optimization}

In this section the EI will be experimentally assessed and compared for the three scenarios defined in Section II-D.

1) No restriction on number of allowed APs: For scenario 1, the resulting optimal solution deploys 9 APs: APs 11, 53, and 55 with an EIRP of $0 \mathrm{dBm}$, APs 2, 3, and 27 with an EIRP of $1 \mathrm{dBm}$, AP 8 with an EIRP of $13 \mathrm{dBm}$, AP 49 with an EIRP of $7 \mathrm{dBm}$ and AP 57 with an EIRP of $11 \mathrm{dBm}$ (see Fig. 2). This shows that APs closer to the users have lower EIRPs. The EI after optimization equals $5.42 \cdot 10^{-10} \mathrm{~W} / \mathrm{kg}$ during 1 hour, versus $2.17 \cdot 10^{-8} \mathrm{~W} / \mathrm{kg}$ for the reference configuration, a reduction by $97.5 \%$ (see Fig. 5). Fig. 6a shows the contributions to the EI of each of the 13 individual wireless users, for both configurations. The exposure reduction varies between a factor 15 at location 46 and a factor 139 at location 24 . Location 46 is relatively far away from the APs 3 and 7 in the reference configuration, keeping the exposure low and thus also the possible reduction. Location 24 is close to AP 3 with EIRP $20 \mathrm{dBm}$ in the reference configuration, so a large reduction is indeed obtained by optimizing the active APs and their EIRP. Fig. 6a also shows that the spatial variation on the exposure of each of the individuals is reduced in the optimized network: the coefficient of variation reduces from 55 to $40 \%$. Importantly, with respect to QoS, it is easily shown that the reference configuration fails to deliver the required capacity. AP 3 connects all 13 users. However, based on the duty cycle assumption of $10.69 \%$ per user for the considered usage (i.e., Youtube 1080p) and the maximal theoretical duty cycle of $69.83 \%$ at 54 Mbps [19], at most 6 users can be appropriately served. It is shown that the presented solution effectively tackles this capacity problem: no AP connects more than 3 users. It can therefore be concluded that the optimized scenario not only causes a lower EI, but also provides a better QoS for this multi-user scenario. For scenario 2, the optimal configuration uses 10 APs: APs 2, 10, 51, 53, 55, 56 with an EIRP of $0 \mathrm{dBm}$, APs 3 and 6 with an EIRP of $1 \mathrm{dBm}$, AP 8 with an EIRP of $2 \mathrm{dBm}$, and AP 4 with an EIRP of $3 \mathrm{dBm}$ (see Fig. 2). The EI after optimization equals $1.082 \cdot 10^{-6} \mathrm{~W} / \mathrm{kg}$ during 8 hours, versus $1.083 \cdot 10^{-6} \mathrm{~W} / \mathrm{kg}$ for the reference configuration, a reduction by only $0.07 \%$ (see Fig. 5). The reason for this negligible reduction is the dominance of the UL contributions to the EI for each of the users, which follows from the absence of power control in WiFi, where the uplink power is fixed at (mostly) $20 \mathrm{dBm}$. This also 


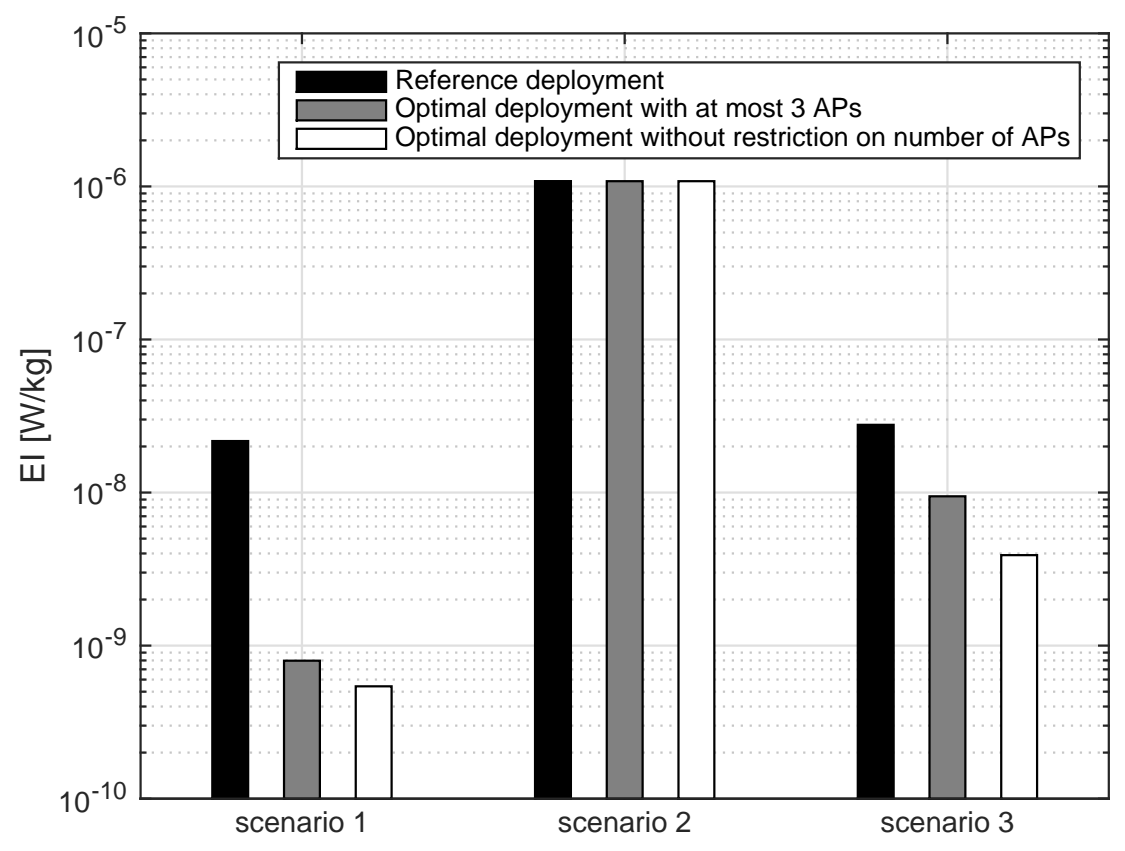

Fig. 5: Exposure Index EI [W/kg] for the three scenarios for the reference deployment, the optimal deployment with at most three APs, and the deployment without restriction on the number of APs.

causes each of the users to be equally exposed, irrespective of their location with respect to the APs: the individual average SAR values lie between $1.0812 \cdot 10^{-6}$ at location 27 and $1.0838 \cdot 10^{-6} \mathrm{~W} / \mathrm{kg}$ at location 34 . On average over all users, the EI contributions of the own uplink, other users' uplink and the APs' downlink equal $99.805 \%, 0.193 \%$, and $0.002 \%$, respectively. Given the low duty cycles, no capacity problems occur. The optimization algorithm efficiently reduces the DL exposure, from $7.32 \cdot 10^{-11} \mathrm{~W} / \mathrm{kg}$ to $1.56 \cdot 10^{-11} \mathrm{~W} / \mathrm{kg}$. However, its contribution to the total EI is negligible in this scenario and therefore, the optimization is not visible in Fig. 7, which shows the evolution of the total EI for the three scenarios.

The optimal configuration in scenario 3 consists of 12 APs with an EIRP of $0 \mathrm{dBm}$ : APs 11, 13, 18, 20, 26, 43, 46, 47, 50, 52, 54, and 57 (see Fig. 2). The EI after optimization equals $3.90 \cdot 10^{-9} \mathrm{~W} / \mathrm{kg}$ during 8 hours, versus $2.77 \cdot 10^{-8} \mathrm{~W} / \mathrm{kg}$ for the reference configuration, a reduction by $86 \%$ (see Fig. 5). Although in scenario 3, the total EI becomes 278 times smaller compared to scenario 2 thanks to uplink power control, the own uplink power still remains the main contributor to the EI: on average over all users, the EI contributions of the own uplink, other users' uplink and the APs' downlink equal $3.856 \cdot 10^{-9}$, $8.895 \cdot 10^{-12}$, or $2.971 \cdot 10^{-12}$, or $99.01 \%, 0.23 \%$, and $0.76 \%$ of the total EI, respectively. Power control in scenario 3 also causes the EI contribution of other users' uplink to become smaller than the APs' downlink contribution, while this was not the case in scenario 2. Individual average SAR values range from $1.45 \cdot 10^{-9} \mathrm{~W} / \mathrm{kg}$ at location 31 to $6.99 \cdot 10^{-9} \mathrm{~W} / \mathrm{kg}$ at location 22. Again, no capacity problems occur.

2) Maximally three APs: Since all the of the presented optimal solutions require the deployment of a large number of access points (at least 9), the algorithm is also run with the maximal number of APs set to three, as in the reference configuration. For scenario 1, the resulting optimal solution deploys AP 3 with an EIRP of $4 \mathrm{dBm}$, AP 39 with an EIRP of $11 \mathrm{dBm}$, and AP 53 with an EIRP of $0 \mathrm{dBm}$. The EI after optimization equals $7.97 \cdot 10^{-10} \mathrm{~W} / \mathrm{kg}$ during 1 hour, versus $2.17 \cdot 10^{-8} \mathrm{~W} / \mathrm{kg}$ for the reference configuration, still a reduction by $96.3 \%$. Fig. 5 indeed shows that only three APs suffice to obtain an EI close to the deployment without restriction on the number of APs: compared to the optimal solution with 9 APs, the EI increases by only a factor 1.47; the coefficient of variation also equals $40 \%$. Fig. $6 \mathrm{a}$ also shows the contributions to the EI of each of the 13 individual wireless users in the optimized configuration with three APs, where reductions vary between a factor 13 at location 25 and a factor 78 at location 24. Thanks to the algorithm, the solution with 3 APs now also satisfies the capacity requirement: APs 3,39, and 53 connect 3, 6, and 4 users respectively.

For scenario 2, the following 3 APs are deployed: AP 4 with an EIRP of $5 \mathrm{dBm}$, AP 8 with an EIRP of $7 \mathrm{dBm}$, and AP 12 with an EIRP of $0 \mathrm{dBm}$. Just as for the optimized configuration with an unlimited amount of APs, the resulting EI is $1.082 \cdot 10^{-6} \mathrm{~W} / \mathrm{kg}$ during 8 hours (see Fig. 5). Here, the EI contribution of the downlink is $0.003 \%$ vs $0.002 \%$ in the fully optimized scenario, a negligible difference. The optimization algorithm again efficiently reduces the DL exposure, from $3.63 \cdot 10^{-10} \mathrm{~W} / \mathrm{kg}$ after the first generation to $3.77 \cdot 10^{-11} \mathrm{~W} / \mathrm{kg}$ after 30 generations.

In scenario 3, a configuration with $3 \mathrm{APs}$ (AP 11 at $1 \mathrm{dBm}$, AP 20 at $4 \mathrm{dBm}$, and AP 47 at $3 \mathrm{dBm}$ ) is able to deliver an EI of 


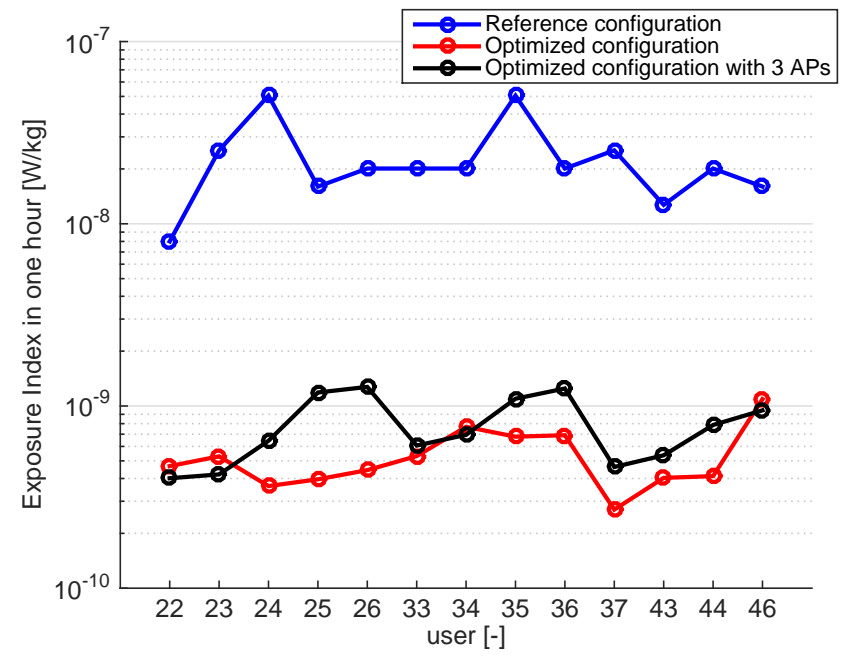

(a) Scenario 1

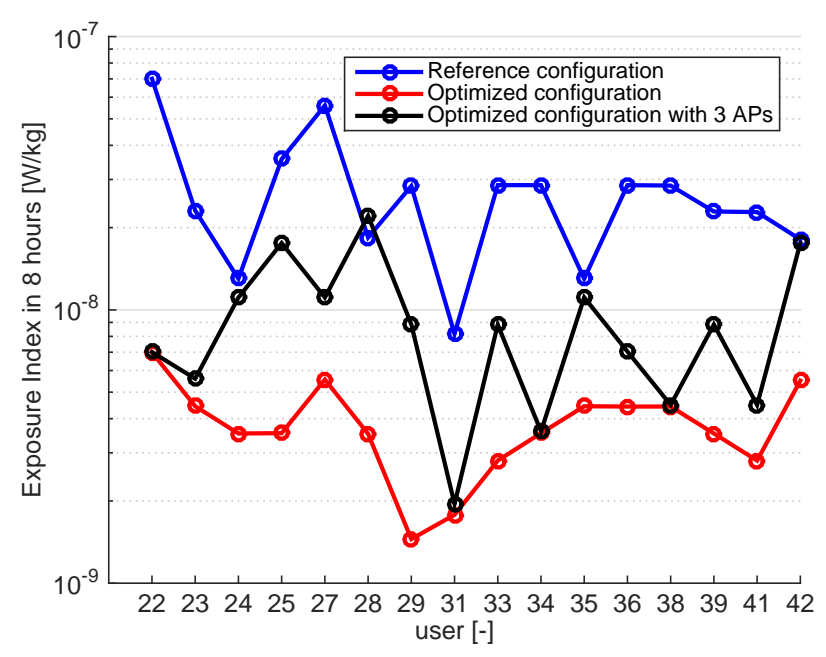

(b) Scenario 3

Fig. 6: EI contribution per user location for the reference configuration and for optimized configurations (with and without a limit to 3 APs).

$9.45 \cdot 10^{-9} \mathrm{~W} / \mathrm{kg}$ during 8 hours. This is a reduction of $66 \%$ with respect to the reference configuration $\left(2.77 \cdot 10^{-8} \mathrm{~W} / \mathrm{kg}\right), \mathrm{vs}$. a reduction by $86 \%$ for the fully optimized configuration with 12 APs (see Fig. 5). Fig. 6b also shows the spatial variation on the exposure of each of the individuals: coefficients of variation on the EI are 57\%, 59\%, and 36\% for the reference configuration, the optimized configuration with 3 APs, and the optimized configuration without restriction on the amount of APs. This shows that for uplink scenarios with power control, more APs are required to provide a more homogeneous individual exposure.

\section{Algorithm performance}

Fig. 7 shows the convergence of the GA for the three scenarios. After 30 generations, an optimum is obtained for all three scenarios. The total runtimes are displayed in Table III. Scenario 1 has a lower runtime than scenarios 2 and 3, since it is a downlink-only scenario. Scenario 3 has a longer runtime than scenario 2, due to the extra calculations related to power control. When the maximal amount of APs is limited to three, runtimes are reduced compared to deployments without limitations on the amount of APs: by $65 \%, 55 \%$, and $65 \%$ for scenarios 1, 2, and 3, respectively. All optimizations are performed on a desktop computer with an Intel Core i5-2400 CPU processor @ 3.10 GHz, with 8 GB RAM.

TABLE III: Runtime for the different scenarios, for the optimization with and without a limit to three APs.

\begin{tabular}{|r|r|r|}
\hline runtime(s) & $\leq$ 3 APs & unlimitedAPs \\
\hline \hline Scenario1 & 91 & 258 \\
\hline Scenario2 & 214 & 475 \\
\hline Scenario3 & 285 & 815 \\
\hline
\end{tabular}

\section{CONCLUSION}

A genetic algorithm has been presented to minimize the exposure index of a set of users within a certain time duration. It is the first algorithm that assesses and optimizes the index based on real-life experimental data. The output of the algorithm is based on the usage and load of the network, considers all exposure sources (downlink, own uplink, other users' uplink) in an experimental way, and accounts for realistic duty cycles. The algorithm is here tested by applying it inside an indoor WiFi testbed for three different scenarios. It is shown that the algorithm successfully reduces the EI in a downlink-only scenario by 97.5\%. In a regular scenario with both downlink and uplink, the network layout has no significant influence on the EI under the absence of uplink power control. This is due to the high default WiFi uplink powers, causing the own uplink contribution to the total EI to be dominant over the other contributions (downlink and uplink of other users). The advances that have recently been made in the deployment of WiFi uplink power control, could in the future clearly reduce the EI. This paper shows that introducing power control reduces the EI by a factor 278 for the same scenario and the same QoS. The algorithm always converges within 30 generations, corresponding to time durations between 91 and $815 \mathrm{~s}$, depending on the input scenario. In the future, the algorithm could be coupled to an electronic meeting scheduler for a guaranteed QoS with a minimal RF exposure for all people attending the meeting. It is also applicable to outdoor networks and can easily be extended to other 


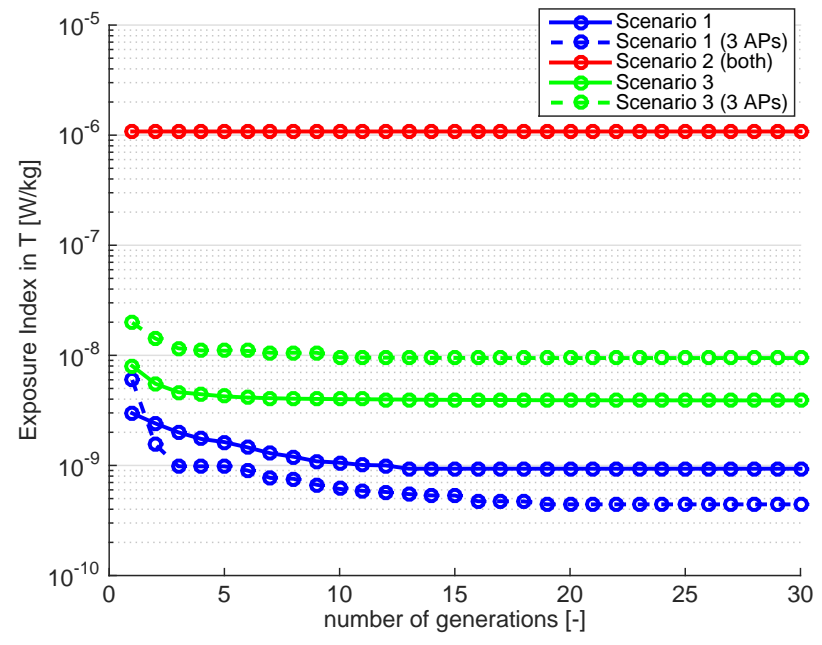

Fig. 7: Evolution of EI contribution as a function of the number of generations, for the different scenarios with and without a limit to 3 APs.

telecommunication networks. A further extension consists of equipping each AP with a module that measures the network usage in real-time and communicates with the developed algorithm to create a cognitive system that dynamically adapts the network layout based on real-time data.

\section{ACKNOWLEDGMENT}

This paper reports work undertaken in the context of the project LEXNET. LEXNET is a project supported by the European Commission in the 7th Framework Programme (GA n318273). For further information, please visit www.lexnet-project.eu.

\section{REFERENCES}

[1] ICNIRP, "Guidelines for limiting exposure to time-varying electric, magnetic, and electromagnetic fields (up to $300 \mathrm{GHz}$ )," Health Physics, vol. 74, no. 4, pp. 494-522, Apr. 1998.

[2] K. R. Foster, "Radiofrequency exposure from wireless LANs using Wi-Fi technology," Health Physics, vol. 92, pp. 280 - $289,2007$.

[3] W. Joseph, P. Frei, M. Roösli, G. Thuróczy, P. Gajsek, T. Trcek, J. Bolte, G. Vermeeren, E. Mohler, P. Juhsz, V. Finta, and L. Martens, "Comparison of personal radio frequency electromagnetic field exposure in different urban areas across Europe." Environmental Research, vol. 110, no. 7, pp. 658 $663,2010$.

[4] P. Frei, E. Mohler, A. Bürgi, J. Fröhlich, G. Neubauer, C. Braun-Fahrländer, and M. Roosli, "A prediction model for personal radio frequency electromagnetic field exposure," Science of The Total Environment, vol. 408, no. 1, pp. 102 - 108, 2009.

[5] A. Boursianis, P. Vanias, and T. Samaras, "Measurements for assessing the exposure from 3G femtocells," Radiation Protection Dosimetry), vol. 150, no. 2 , pp. $158-167,2012$.

[6] D. Plets, W. Joseph, S. Aerts, K. Vanhecke, and L. Martens, "Prediction and Comparison of Downlink Electric-Field and Uplink Localized SAR Values for Realistic Indoor Wireless Network Planning," Radiation Protection Dosimetry, February 2014.

[7] A. Peyman, C. Gabriel, E. H. Grant, G. Vermeeren, and L. Martens, "Variation of the dielectric properties of tissues with age: the effect on the values of sar in children when exposed to walkietalkie devices," Physics in Medicine and Biology, vol. 54, no. 2, pp. 227-241, 2009. [Online]. Available: http://stacks.iop.org/0031-9155/54/i=2/a=004

[8] G. Vermeeren, W. Joseph, and L. Martens, "Whole-body sar in spheroidal adult and child phantoms in realistic exposure environment," ELECTRONICS LETTERS, vol. 44, no. 13, pp. 790-791, 2008. [Online]. Available: http://dx.doi.org/10.1049/el:20080770

[9] A. Christ, M.-C. Gosselin, M. Christopoulou, S. Khn, and N. Kuster, "Age-dependent tissue-specific exposure of cell phone users," Physics in Medicine and Biology, vol. 55, no. 7, pp. 1767-1783, 2010. [Online]. Available: http://stacks.iop.org/0031-9155/55/i=7/a=001

[10] A. Christ, W. Kainz, E. G. Hahn, K. Honegger, M. Zefferer, E. Neufeld, W. Rascher, R. Janka, W. Bautz, J. Chen, B. Kiefer, P. Schmitt, H.-P. Hollenbach, J. Shen, M. Oberle, D. Szczerba, A. Kam, J. W. Guag, and N. Kuster, "The virtual familydevelopment of surface-based anatomical models of two adults and two children for dosimetric simulations," Physics in Medicine and Biology, vol. 55, no. 2, p. N23, 2010. [Online]. Available: http://stacks.iop.org/0031-9155/55/i=2/a=N01

[11] W. Joseph, P. Frei, M. Roösli, G. Vermeeren, J. Bolte, G. Thuróczy, P. Gajsek, T. Trcek, E. Mohler, P. Juhasz, V. Finta, and L. Martens, "Between-country comparison of whole-body SAR from personal exposure data in urban areas," Bioelectromagnetics, vol. 33, no. 8, pp. 682-694, December 2012.

[12] D. Plets, W. Joseph, K. Vanhecke, G. Vermeeren, J. Wiart, S. Aerts, N. Varsier, and L. Martens, "Joint minimization of uplink and downlink whole-body dose in indoor wireless networks," BioMed Research International, special issue on Public Health Assessment of Impact of Heterogeneous Wireless Systems in Nonionizing Radiation Exposure, in press.

[13] O. Lauer, P. Frei, M. Gosselin, W. Joseph, M. Roosli, and F. J., "Combining near-and far-field exposure for an organ-specific and whole-body rf-emf proxy for epidemiological research: a reference case." Bioelectromagnetics, vol. 34, no. 5, pp. 366-374, 2013.

[14] Varsier, N., Plets, D., Corre, Y., Vermeeren, G., Joseph, W., Aerts, S., Martens, L. and Wiart, J., "A novel method to assess human population exposure induced by a wireless cellular network." BioElectromagnetics, vol. 36, pp. 451-463, 2015.

[15] D. Plets, W. Joseph, K. Vanhecke, and L. Martens, "Exposure Optimization in Indoor Wireless Networks by Heuristic Network Planning," Progress In Electromagnetic Research (PIER), vol. 139, pp. 445-478, 2013. 
[16] Plets, D., Joseph, W., Aerts, S., Vermeeren, G., Varsier, N., Wiart, J. and Martens, L., “Assessment of contribution of other users to own total whole-body RF absorption in train environment." BioElectromagnetics, vol. 36, pp. 597-602, 2015.

[17] A. Gati, E. Conil, M.-F. Wong, and J. Wiart, "Duality between uplink local and downlink whole-body exposures in operating networks," Electromagnetic Compatibility, IEEE Transactions on, vol. 52, no. 4, pp. 829-836, 2010.

[18] European Commission, "Eu fp7 program, 2014," Website, http://ec.europa.eu/research/fp7/index_en.cfm.

[19] W. Joseph, D. Pareit, G. Vermeeren, D. Naudts, L. Verloock, L. Martens, and I. Moerman, "Determination of the duty cycle of WLAN for realistic radio frequency electromagntic field exposure assessment," Progress in Biophysics \& Molecular Biology, October 2012.

[20] Lexnet consortium, "Lexnet D2.8: Global Wireless Exposure Metric Definition," Tech. Rep. [Online]. Available: http://cordis.europa.eu/docs/projects/cnect/3/318273/080/deliverables/001-LEXNETWP2D28GlobalwirelessexposuremetricdefAres20155347928.pdf

[21] D. Plets, W. Joseph, K. Vanhecke, E. Tanghe, and L. Martens, "Coverage Prediction and Optimization Algorithms for Indoor Environments," EURASIP Journal on Wireless Communications and Networking, Special Issue on Radio Propagation, Channel Modeling, and Wireless, Channel Simulation Tools for Heterogeneous Networking Evaluation, vol. 1, 2012. [Online]. Available: http://jwcn.eurasipjournals.com/content/2012/1/123

[22] W. Choi, H. Lim, and A. Sabharwal, "Power-controlled medium access control protocol for full-duplex wifi networks," IEEE Transactions on Wireless Communications, vol. 14, no. 7, pp. 3601-3613, July 2015.

[23] T. Huehn and C. Sengul, "Practical power and rate control for wifi," in 2012 21st International Conference on Computer Communications and Networks (ICCCN), July 2012, pp. 1-7.

[24] S. R. Saunders, Antennas and propagation for wireless communication systems. New York, NY.: John Wiley \& Sons Inc., 1999. 